\title{
Search for trinucleon decay in the Majorana Demonstrator
}

S. I. Alvis ${ }_{9}^{1}$ I. J. Arnquist, ${ }^{2}$ F. T. Avignone III, ${ }^{3,4}$ A. S. Barabash,${ }^{5}$ C. J. Barton, ${ }^{6}$ V. Basu, ${ }^{7}$ F. E. Bertrand, ${ }^{4}$ B. Bos,${ }^{8}$ V. Brudanin, ${ }^{9}$ M. Busch, ${ }^{11,11}$ M. Buuck, T. S. Caldwell, ${ }^{12,11}$ Y-D. Chan,${ }^{13}$ C. D. Christofferson, ${ }^{8}$ P.-H. Chu, ${ }^{14}$ C. Cuesta, ${ }^{1,}$ J. A. Detwiler, ${ }^{1}$ Yu. Efremenko, ${ }^{15,4}$ H. Ejiri, ${ }^{16}$ S. R. Elliott, ${ }^{14}$ T. Gilliss, ${ }^{2,11}$ G. K. Giovanetti ${ }^{17}$ M. P. Green, ${ }^{18,11,4}$ J. Gruszko, ${ }^{19}$ I. S. Guinn, ${ }^{1}$ V. E. Guiseppe, ${ }^{3}$ C. R. Haufe, ${ }^{12,11}$ R. J. Hegedus, ${ }^{12,11}$ L. Hehn, ${ }^{13}$ R. Henning, ${ }^{12,11}$ D. Hervas Aguilar, ${ }^{12,11}$ E. W. Hoppe, ${ }^{2}$ M. A. Howe, ${ }^{12,11}$ K. J. Keeter, ${ }^{20}$ M. F. Kidd, ${ }^{21}$ S. I. Konovalov, ${ }^{5}$ R. T. Kouzes, ${ }^{2}$ A. M. Lopez, ${ }_{7}^{15}$ R. D. Martin, ${ }^{7}$ R. Massarczyk,${ }^{14}$ S. J. Meijer, ${ }^{1,11}$ S. Mertens ${ }^{22,23}$ J. Myslik, ${ }_{11}^{13}$ G. Othman, ${ }^{12,11}$ W. Pettus, ${ }^{1}$ A. Piliounis, ${ }^{7}$ A. W. P. Poon, ${ }^{13}$ D. C. Radford, ${ }^{4}$ J. Rager, ${ }^{12,11}$ A. L. Reine, ${ }^{12,11}$ K. Rielage, ${ }^{14}$ N. W. Ruof, ${ }^{1}$ B. Shanks, ${ }^{4}$ M. Shirchenko, ${ }^{9}$ D. Tedeschi, ${ }^{3}$ R. L. Varner, ${ }^{4}$ S. Vasilyev, ${ }^{9}$ B. R. White, ${ }^{14}$ J. F. Wilkerson,,${ }^{12,11,4}$ C. Wiseman, ${ }^{1}$ W. Xu, ${ }^{6}$ E. Yakushev, ${ }^{9}$ C.-H. Yu, ${ }^{4}$ V. Yumatov ${ }^{5}$ I. Zhitnikov, ${ }^{9}$ and B. X. Zhu ${ }^{14}$

\section{(MAJORAna Collaboration)}

\author{
${ }^{1}$ Center for Experimental Nuclear Physics and Astrophysics, \\ and Department of Physics, University of Washington, Seattle, Washington 98195, USA \\ ${ }^{2}$ Pacific Northwest National Laboratory, Richland, Washington 99354, USA \\ ${ }^{3}$ Department of Physics and Astronomy, University of South Carolina, Columbia, South Carolina 29208, USA \\ ${ }^{4}$ Oak Ridge National Laboratory, Oak Ridge, Tennessee 37830, USA \\ ${ }^{5}$ National Research Center "Kurchatov Institute" Institute for Theoretical and Experimental Physics, \\ Moscow 117218, Russia \\ ${ }^{6}$ Department of Physics, University of South Dakota, Vermillion, South Dakota 57069, USA \\ ${ }^{7}$ Department of Physics, Engineering Physics and Astronomy, Queen's University, \\ Kingston, Ontario K7L 3N6, Canada \\ ${ }^{8}$ South Dakota School of Mines and Technology, Rapid City, South Dakota 57701, USA \\ ${ }^{9}$ Joint Institute for Nuclear Research, Dubna 141980, Russia \\ ${ }^{10}$ Department of Physics, Duke University, Durham, North Carolina 27708, USA \\ ${ }^{11}$ Triangle Universities Nuclear Laboratory, Durham, North Carolina 27708, USA \\ ${ }^{12}$ Department of Physics and Astronomy, University of North Carolina, \\ Chapel Hill, North Carolina 27514, USA \\ ${ }^{13}$ Nuclear Science Division, Lawrence Berkeley National Laboratory, Berkeley, California 94720, USA \\ ${ }^{14}$ Los Alamos National Laboratory, Los Alamos, New Mexico 87545, USA \\ ${ }^{15}$ Department of Physics and Astronomy, University of Tennessee, Knoxville, Tennessee 37916, USA \\ ${ }^{16}$ Research Center for Nuclear Physics, Osaka University, Ibaraki, Osaka 567-0047, Japan \\ ${ }^{17}$ Department of Physics, Princeton University, Princeton, New Jersey 08544, USA \\ ${ }^{18}$ Department of Physics, North Carolina State University, Raleigh, North Carolina 27695, USA \\ ${ }^{19}$ Department of Physics, Massachusetts Institute of Technology, Cambridge, Massachusetts 02139-4307, USA \\ ${ }^{20}$ Department of Physics, Black Hills State University, Spearfish, South Dakota 57799, USA \\ ${ }^{21}$ Tennessee Tech University, Cookeville, Tennessee 38505, USA \\ ${ }^{22}$ Max-Planck-Institut für Physik, München 80805, Germany \\ ${ }^{23}$ Physik Department, Technische Universität, München 85748, Germany
}

(Received 3 December 2018; published 12 April 2019)

\begin{abstract}
The MAJORAnA DemOnSTRATOR is an ultra-low-background experiment searching for neutrinoless double-beta decay in ${ }^{76} \mathrm{Ge}$. The heavily shielded array of germanium detectors, placed nearly a mile underground at the Sanford Underground Research Facility in Lead, South Dakota, also allows searches for new exotic physics. We present the first limits for trinucleon decay-specific modes and invisible decay modes for Ge isotopes. We find a half-life limit of $4.9 \times 10^{25} \mathrm{yr}$ for the decay ${ }^{76} \mathrm{Ge}(\mathrm{ppn}) \rightarrow{ }^{73} \mathrm{Zn} e^{+} \pi^{+}$and $4.7 \times 10^{25} \mathrm{yr}$ for the decay ${ }^{76} \mathrm{Ge}(\mathrm{ppp}) \rightarrow{ }^{73} \mathrm{Cu} e^{+} \pi^{+} \pi^{+}$. The half-life limit for the invisible triproton decay mode of ${ }^{76} \mathrm{Ge}$ was found to be $7.5 \times 10^{24} \mathrm{yr}$.
\end{abstract}

DOI: 10.1103/PhysRevD.99.072004

*Present address: Centro de Investigaciones Energéticas, Medioambientales y Tecnológicas, CIEMAT 28040, Madrid, Spain.

Published by the American Physical Society under the terms of the Creative Commons Attribution 4.0 International license. Further distribution of this work must maintain attribution to the author(s) and the published article's title, journal citation, and DOI. Funded by SCOAP ${ }^{3}$. 


\section{INTRODUCTION}

The conservation of the number of baryons $(B)$ in any reaction is an empirical symmetry of the Standard Model that is not the result of any fundamental principle. Hence, there are numerous reasons to consider its violation $(\not \not B)$. Theories that unify the strong and electroweak forces naturally include $\not B$. It is expected that quantum gravity theories will violate $B$ or any similar global symmetry. Theories with extra dimensions permit particle disappearance, and nucleon decay can be induced via interactions with dark matter as manifest in asymmetric dark matter theories. $\not B$ is also one of the Sakharov requirements to explain the matter-antimatter asymmetry of the Universe. These topics and the possibility of $\not B$ are reviewed in Ref. [1] and references therein. Therefore, the scientific motivation for studying $\not B$ is compelling. The breadth of model possibilities is very broad, however, indicating that many complementary search techniques could help elucidate the question.

The Standard Model with small neutrino masses has an anomaly-free $Z_{6}$ symmetry that acts as discrete $B$ [2]. In this model $\Delta B=1$ or 2 processes are forbidden, but $\Delta B=$ 3 transitions can arise due to a dimension 15 operator. When undergoing a $\Delta B=3$ trinucleon decay, three baryons disappear from the nucleus, frequently leaving an isotope that is unstable. Previous searches in Xe isotopes [3,4] and ${ }^{127}$ I [5] looked for invisible decay channels assuming no observation of the initial trinucleon decay or disappearance. Only the decay of the unstable product was sought as evidence for the process. Other groups considered invisible $\Delta B=2$ decays with limits reported in Refs. [6-13]. Results for $\Delta B=2,3$ decays from the Majorana Demonstrator are presented here for invisible channels and for decay-specific modes.

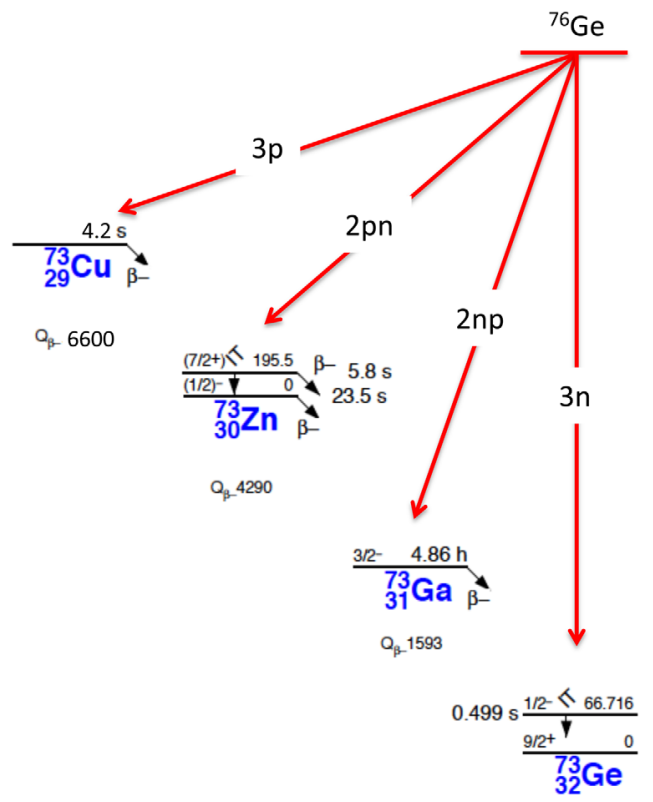

FIG. 1. The ${ }^{76} \mathrm{Ge}$ decay scheme. Figure adapted from Ref. [14].
The dominant decay modes for $\Delta B=3$ are given in Ref. [2] as

$$
\begin{aligned}
& p p p \rightarrow e^{+} \pi^{+} \pi^{+} \\
& p p n \rightarrow e^{+} \pi^{+} \\
& p n n \rightarrow e^{+} \pi^{0} \\
& n n n \rightarrow \bar{\nu} \pi^{0} .
\end{aligned}
$$

The resulting daughter nuclei for these processes in ${ }^{76} \mathrm{Ge}$ are displayed in Fig. 1. Typical modes of decay for $\Delta B=2$ are

$$
\begin{aligned}
& p p \rightarrow \pi^{+} \pi^{+} \\
& p n \rightarrow \pi^{0} \pi^{+} \\
& n n \rightarrow \pi^{+} \pi^{-}, \pi^{0} \pi^{0} .
\end{aligned}
$$

\section{THE MAJORANA DEMONSTRATOR}

The Majorana Demonstrator described in detail in Refs. $[15,16]$ is located at a depth of $4850 \mathrm{ft}$ at the Sanford Underground Research Facility in Lead, South Dakota [17]. In addition to its primary goal of searching for neutrinoless double-beta decay, its ultra-low-background configuration permits additional physics studies including searches for dark matter [18], axions, and exotic physics (e.g. Ref. [19]). Two modules contain $44.1 \mathrm{~kg}$ of highpurity germanium P-type point-contact detectors, of which $29.7 \mathrm{~kg}$ have $88 \%{ }^{76} \mathrm{Ge}$ enrichment. Fifty-eight detector units are installed in strings of three, four, or five detectors. These strings of detectors are mounted within vacuum cryostats which are shielded from room background by a lead and copper shield. The entire apparatus is contained within a $4-\pi$ cosmic ray veto system $[20,21]$.

The low energy thresholds, excellent energy resolution, reduced electronic noise, and pulse shape characteristics of the P-type point contact detectors [22-25] enable the sensitive double beta decay search. The nucleon decay analyses presented here include data taken from June 2015 until April 2018. Excluding calibration, commissioning data and data taken during intense mechanical work, the analyzed data includes $26.0 \mathrm{~kg}$ yr of enriched exposure and $9.45 \mathrm{~kg}$ yr of natural exposure [26]. The data are divided into data sets referred to as DS0 through DS6 and a detailed description of each set is given in Ref. [16]. All the analyses described here were developed on the data sets published in Ref. [16] (approximately $1 / 3$ of the total) and then executed on the full data sets after unblinding. The data blinding scheme parses the data into open $(25 \%$ of run time) and blind (75\%) partitions [26].

The Demonstrator records every pulse with two digitizer channels with different amplifications to permit studies of the energy spectrum from below $1 \mathrm{keV}$ to above $10 \mathrm{MeV}$. This work analyzes the spectrum from $100 \mathrm{keV}$ to 
saturation (about $11 \mathrm{MeV}$ ). Energy deposits above saturation are recorded within an overflow channel and identified with a dedicated tag.

\section{TRIBARYON DECAY IN GE ISOTOPES}

Due to the enrichment of the Ge in the Demonstrator, the isotope ${ }^{76} \mathrm{Ge}$ has the largest exposure and dominates the sensitivity to $\not B$. Therefore we describe the analysis of the triproton decay channel of ${ }^{76} \mathrm{Ge}$ in some detail here as an example. All searched-for signatures are summarized in Table I. We report results for decays of all Ge isotopes present in the DemONSTRATOR, ${ }^{70,72,73,74,76} \mathrm{Ge}$.

The two analyses described here, invisible decay modes and decay-specific modes, are similar but have minor differences arising from the relative signature efficiency optimization. The signature for an invisible decay mode is the sequence of decays of the resulting unstable daughter, ignoring any potential signature from the initial disappearance of the nucleons. In the decay-specific mode searches, the decays of the unstable daughter nuclei are sought following an initial signature from the $\not \beta$ decay. For the DEMONSTRATOR the most sensitive channel, in both the decay-specific and invisible modes, is the triproton decay of ${ }^{76} \mathrm{Ge}$ to ${ }^{73} \mathrm{Cu}$. The resulting ${ }^{73} \mathrm{Cu}$ isotope is $\beta$ unstable with a $4.2 \mathrm{~s}$ half-life and a Q-value of $6.6 \mathrm{MeV}$. Its daughter ${ }^{73} \mathrm{Zn}$ is also $\beta$ unstable with a $23.5 \mathrm{~s}$ half-life and a Q-value of $4.3 \mathrm{MeV}$. Since the count rate is very low in the DEMONSTRATOR above the two-neutrino double-beta decay end point ( $2 \mathrm{MeV})$, a signature of two $\beta$ decay candidates occurring within five half-lives (117 s) of one another, each above $2 \mathrm{MeV}$, has very little background.

We chose a high-efficiency, five half-life time window between events to select candidate delayed coincidences. The average time between events with energy greater than $100 \mathrm{keV}$ in a typical Demonstrator detector is $\approx 3 \mathrm{~h}$ and the decays of some long-lived isotopes were not considered due to potential accidental coincidence background. To keep the expected accidental background below 1 count with our time cut criterion, only isotopes with a half-life of $<40 \mathrm{~m}$ were considered. This excluded consideration of the dinucleon decays of ${ }^{74} \mathrm{Ge}$, for example. In practice, the longest coincidence window we considered was $105 \mathrm{~m}$, corresponding to the $21 \mathrm{~m}$ half-life of ${ }^{70} \mathrm{Ga}$.

\section{INVISIBLE DECAY PROCESSES}

To select candidate events for invisible decays, we remove events in coincidence with the muon veto and those that fail the delayed-charge recovery (DCR) cut. The use of the DCR cut for this subset of the analysis reduces background due to alpha particles originating from near the detector surface. We do not reject multidetector events or those waveforms symptomatic of multisite events as some $\gamma \mathrm{s}$ might deposit energy in multiple locations. All these cuts are described in detail in Ref. [16] and references therein. We then require energy and timing correlations between successive events within a lone detector to match a particular decay candidate. (See Table I.)

TABLE I. A summary of the signatures of each decay channel for which the MAJORANA DEMONSTRATOR has sensitivity, specifying the energy and timing requirements for the successive decays. The invisible decay mode signatures are composed of two successive decays and hence have two energy constraints and one time constraint. The decay-mode specific signatures include an initial saturated event (not listed here), followed by one or more decays at the energies listed below. N.A. is shorthand for not applicable.

\begin{tabular}{lcccc}
\hline \hline Decay mode & \multicolumn{1}{c}{$\tau_{1}$} & $E_{1}$ & $\tau_{2}$ & $E_{2}$ \\
\hline${ }^{76} \mathrm{Ge}(\mathrm{ppp}) \rightarrow{ }^{73} \mathrm{Cu} \rightarrow{ }^{73} \mathrm{Zn}$ & N.A. & Invisible decay modes & & \\
${ }^{76} \mathrm{Ge}(\mathrm{pp}) \rightarrow{ }^{74} \mathrm{Zn} \rightarrow{ }^{74} \mathrm{Ga}$ & N.A. & $(2.0,6.6) \mathrm{MeV}$ & $\Delta T<117 \mathrm{~s}$ & $(2.0,4.3) \mathrm{MeV}$ \\
${ }^{74} \mathrm{Ge}(\mathrm{ppp}) \rightarrow{ }^{71} \mathrm{Cu} \rightarrow{ }^{71} \mathrm{Zn}$ & N.A. & $(2.0,4.6) \mathrm{MeV}$ & $\Delta T<40 \mathrm{~m}$ & $(2.0,5.4) \mathrm{MeV}$ \\
& & Decay-specific modes & & $(2.0,2.8) \mathrm{MeV}$ \\
& $\Delta T<21 \mathrm{~s}$ & $(0.1,6.6) \mathrm{MeV}$ & $\Delta T<117 \mathrm{~s}$ & $(0.1,4.3) \mathrm{MeV}$ \\
${ }^{76} \mathrm{Ge}(\mathrm{ppp}) \rightarrow{ }^{73} \mathrm{Cu} e^{+} \pi^{+} \pi^{+}$ & $\Delta T<117 \mathrm{~s}$ & $(0.1,4.3) \mathrm{MeV}$ & N.A. & N.A. \\
${ }^{76} \mathrm{Ge}(\mathrm{ppn}) \rightarrow{ }^{73} \mathrm{Zn} e^{+} \pi^{+}$ & $\Delta T<4.5 \mathrm{~m}$ & $(0.1,2.3) \mathrm{MeV}$ & $\Delta T<40 \mathrm{~m}$ & $(0.1,5.4) \mathrm{MeV}$ \\
${ }^{76} \mathrm{Ge}(\mathrm{pp}) \rightarrow{ }^{74} \mathrm{Zn} \pi \pi^{+} \pi^{+}$ & $(0.1,5.4) \mathrm{MeV}$ & N.A. & N.A. \\
${ }^{76} \mathrm{Ge}(\mathrm{pn}) \rightarrow{ }^{74} \mathrm{Ga} \pi^{0} \pi^{+}$ & $\Delta T<40 \mathrm{~m}$ & $(0.1,4.6) \mathrm{MeV}$ & $\Delta T<12.5 \mathrm{~m}$ & $(0.1,2.8) \mathrm{MeV}$ \\
${ }^{74} \mathrm{Ge}(\mathrm{ppp}) \rightarrow{ }^{71} \mathrm{Cu} e^{+} \pi^{+} \pi^{+}$ & $\Delta T<100 \mathrm{~s}$ & $(0.1,2.8) \mathrm{MeV}$ & N.A. & N.A. \\
${ }^{74} \mathrm{Ge}(\mathrm{ppn}) \rightarrow{ }^{71} \mathrm{Zn} e^{+} \pi^{+}$ & $\Delta T<12.5 \mathrm{~m}$ & $(0.1,6.6) \mathrm{MeV}$ & N.A. & N.A. \\
${ }^{73} \mathrm{Ge}(\mathrm{ppp}) \rightarrow{ }^{70} \mathrm{Cu} e^{+} \pi^{+} \pi^{+}$ & $\Delta T<25 \mathrm{~s}$ & $(0.1,1.7) \mathrm{MeV}$ & N.A. & N.A. \\
${ }^{73} \mathrm{Ge}(\mathrm{pnn}) \rightarrow{ }^{70} \mathrm{Ga} e^{+} \pi^{0}$ & $\Delta T<105 \mathrm{~m}$ & $(0.1,2.8) \mathrm{MeV}$ & N.A. & N.A. \\
${ }^{73} \mathrm{Ge}(\mathrm{pp}) \rightarrow{ }^{71} \mathrm{Zn} \pi^{+} \pi^{+}$ & $\Delta T<12.5 \mathrm{~m}$ & $(0.1,2.7) \mathrm{MeV}$ & N.A. & N.A. \\
${ }^{72} \mathrm{Ge}(\mathrm{ppp}) \rightarrow{ }^{69} \mathrm{Cu} e^{+} \pi^{+} \pi^{+}$ & $\Delta T<15 \mathrm{~m}$ & $(0.1,1.7) \mathrm{MeV}$ & N.A. & N.A. \\
${ }^{72} \mathrm{Ge}(\mathrm{pn}) \rightarrow{ }^{70} \mathrm{Ga} \pi^{0} \pi^{+}$ & $\Delta T<105 \mathrm{~m}$ & $(0.1,4.4) \mathrm{MeV}$ & N.A. & N.A. \\
${ }^{70} \mathrm{Ge}(\mathrm{nnn}) \rightarrow{ }^{67} \mathrm{Ge} \bar{\nu} \pi^{0}$ & $\Delta T<95 \mathrm{~m}$ & & & \\
\hline \hline
\end{tabular}


The total efficiency $\left(\epsilon_{\text {tot }}\right)$ is equal to the product of all the efficiencies due to the time correlation cuts and the energy cuts. For the invisible decay modes, we study signatures with two beta decays. The efficiency of the cut due to the decay of the second beta emitter is referred to as $\epsilon_{\tau 2}$. (Note that $\epsilon_{\tau 1}$ plays no role in the analysis of the invisible decay modes as there is no indicator for the creation of the first nucleus. This is in contrast to the decay-specific modes discussed below.)

For the invisible decay, a GEANT4-based[27] Monte Carlo simulation framework (MAGE) [28,29], was used to study the efficiency of the $\beta / \gamma$ decays depositing energy above the threshold. The decay manager within GEANT4 was used to simulate each isotope decay including branchings to excited states. For each isotope, we generated 1 million events in a detector and constrained the decay chain only to its daughter but no further. Figure 2 shows an example of the simulated energy spectrum of ${ }^{73} \mathrm{Cu}$ and ${ }^{73} \mathrm{Zn}$ decays in the detector. We calculated the efficiency $\left(\epsilon_{E_{1}}\right)$ as the fraction of the events
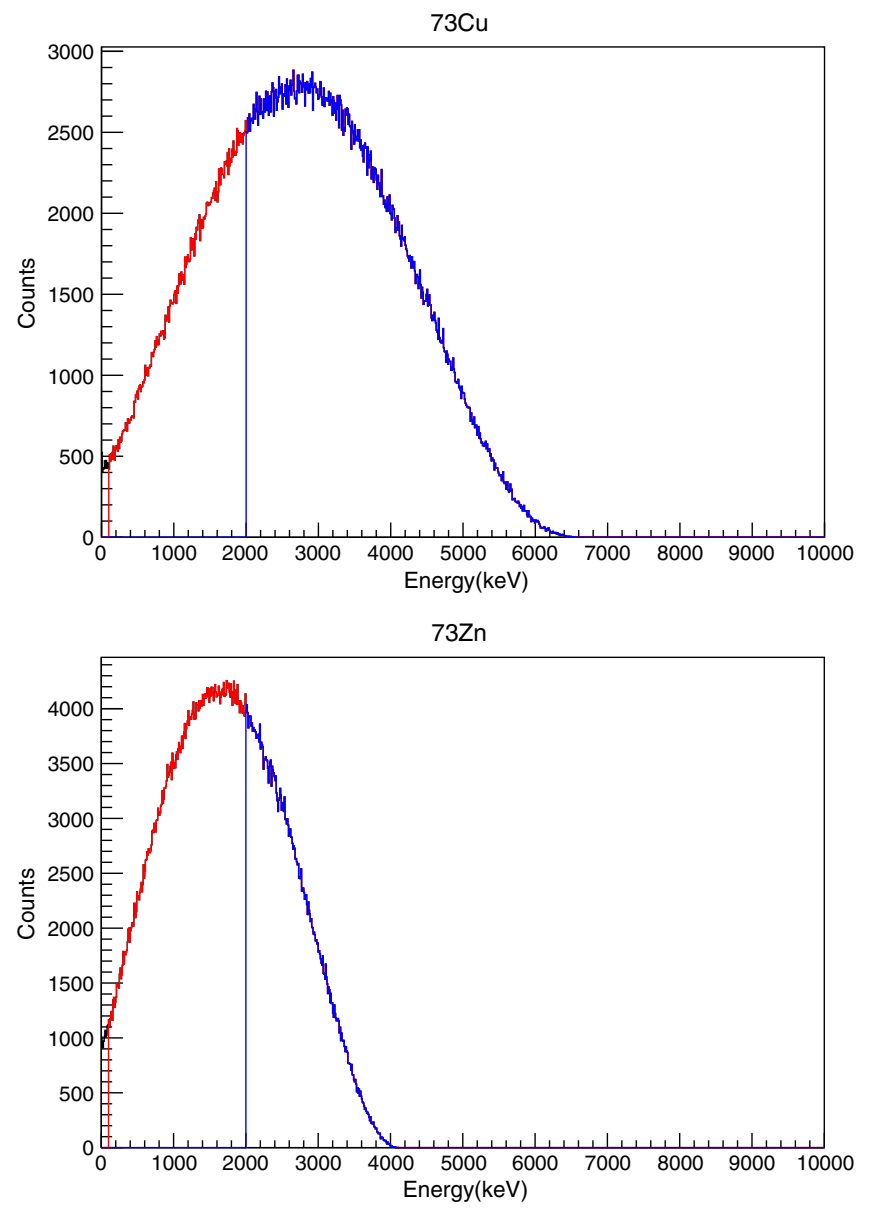

FIG. 2. The simulated energy deposit due to (Top) ${ }^{73} \mathrm{Cu}$ and (Bottom) ${ }^{73} \mathrm{Zn}$. The fraction of the spectrum above $2 \mathrm{MeV}$ for ${ }^{73} \mathrm{Cu}$ decay is $70.7 \%$ and for ${ }^{73} \mathrm{Zn}$ decay is $37.5 \%$. The fraction of the spectrum above $100 \mathrm{keV}$ for ${ }^{73} \mathrm{Cu}$ decay is $99.6 \%$ and for ${ }^{73} \mathrm{Zn}$ decay is $99.0 \%$. with energy larger than $2 \mathrm{MeV}$ deposited. The MAGE simulation framework has been vetted by comparison to MAJORANA ${ }^{228}$ Th calibration [18] and is found to describe the detector response very well. At energies of relevance here above $100 \mathrm{keV}$, the agreement is better than $2 \%$. It is even better if only one detector responds or when the energies are larger than $500 \mathrm{keV}$.

The time cut efficiency takes into account the boundaries of data acquisition periods. We define the efficiencies corresponding to the energy restrictions on the two $\beta$ decays as $\epsilon_{E 1}$ and $\epsilon_{E 2}$ corresponding to the first and second decay, respectively. For the invisible decay modes, $\epsilon_{\text {tot }}=$ $\epsilon_{E 1} \epsilon_{\tau 2} \epsilon_{E 2} \epsilon_{D C R}^{2}$, where $\epsilon_{D C R}$ represents a delayed charge recovery (DCR) waveform cut that rejects $\alpha$ induced signals [30].

The half-life limit $\left(T_{1 / 2}\right)$ is

$$
T_{1 / 2}>\frac{\ln (2) N T \epsilon_{\mathrm{tot}}}{S}
$$

where $N$ is the number of isotopic atoms within the detector active volume and $T$ is the live time in years. We found one such candidate for ${ }^{76} \mathrm{Ge}$ decay and used the FeldmanCousins limit [31] to set an upper limit on the number of events that could be assigned to the process of $S=4.36$ at the $90 \%$ confidence-level half-life limit [Eq. (3)]. The efficiency for this signature $\left(\epsilon_{\text {tot }}=0.257\right)$ includes factors due to the fraction of the beta decays with energy greater than $2 \mathrm{MeV},\left(\epsilon_{E 1}=0.707, \epsilon_{E 2}=0.375\right)$, and the five halflife time restriction $\left(\epsilon_{\tau 2}=0.969\right)$ on the time difference between the two energy deposits, corresponding to the halflife $\tau_{2}$ in this case. In addition, each of the two waveforms must survive the DCR cut. This efficiency $\left(\epsilon_{\mathrm{DCR}} \sim 0.99\right.$ for each waveform) varies from data set to data set but is near this nominal value. We account for the variation in the calculation of the product of efficiency and exposure.

We perform a similar analysis for the invisible diproton decay of ${ }^{76} \mathrm{Ge}$ and the triproton decay of ${ }^{74} \mathrm{Ge}$. Table II lists the two events which can be considered candidates for any of these three invisible decay channels. The half-life limit results are given in Table III. Figure 3 shows the delayed coincidence spectra indicating the low background for these processes once the various cuts are implemented.

TABLE II. The two candidate events for the invisible decays indicating processes to which they correspond. We assume each event is likely to be background for the indicated process when we calculate half-life limits. The ${ }^{76} \mathrm{Ge}(\mathrm{pp})$ and ${ }^{76} \mathrm{Ge}(\mathrm{ppp})$ processes each have one corresponding event. The ${ }^{74} \mathrm{Ge}(\mathrm{ppp})$ process has 2 .

\begin{tabular}{lcccc}
\hline \hline & $\begin{array}{c}E_{1} \\
\text { Event }\end{array}$ & $\begin{array}{c}E_{2} \\
(\mathrm{keV})\end{array}$ & $\begin{array}{c}\tau_{2} \\
\text { CeV) }\end{array}$ & Candidate Process(es) \\
\hline 1 & 4085 & 2164 & $\Delta T=12.9 \mathrm{~s}$ & ${ }^{76} \mathrm{Ge}(\mathrm{ppp}),{ }^{74} \mathrm{Ge}(\mathrm{ppp})$ \\
2 & 2092 & 2353 & $\Delta T=2.7 \mathrm{~m}$ & ${ }^{76} \mathrm{Ge}(\mathrm{pp}),{ }^{74} \mathrm{Ge}(\mathrm{ppp})$ \\
\hline \hline
\end{tabular}


TABLE III. Efficiencies, exposures, signal upper limits and half-life limits for the modes of nucleon decay for the Ge isotopes for which the Demonstrator has an interesting sensitivity. The signal upper limit $(S)$ is the Feldman-Cousins $90 \%$ upper limit $(S)$ given a number of observed candidates. N.A. is shorthand for not applicable.

\begin{tabular}{|c|c|c|c|c|c|c|c|c|c|c|}
\hline Decay mode & $\epsilon_{0}$ & $\epsilon_{\tau 1}$ & $\epsilon_{E 1}$ & $\epsilon_{\tau 2}$ & $\epsilon_{E 2}$ & $\epsilon_{\text {tot }}$ & $\begin{array}{c}\mathrm{NT} \epsilon_{\mathrm{tot}} \\
\left(10^{24} \text { atom yr }\right)\end{array}$ & Candidates & $\begin{array}{c}S \\
\text { (counts) }\end{array}$ & $\begin{array}{c}T_{1 / 2} \\
\left(10^{24} \mathrm{yr}\right)\end{array}$ \\
\hline \multicolumn{11}{|c|}{ Invisible decay modes } \\
\hline${ }^{76} \mathrm{Ge}(\mathrm{ppp}) \rightarrow{ }^{73} \mathrm{Cu}$ & N.A. & N.A. & 0.707 & 0.969 & 0.375 & 0.26 & 47.1 & 1 & 4.36 & 7.5 \\
\hline${ }^{76} \mathrm{Ge}(\mathrm{pp}) \rightarrow{ }^{74} \mathrm{Zn}$ & N.A. & N.A. & 0.004 & 0.969 & 0.367 & 0.002 & 0.28 & 1 & 4.36 & 0.05 \\
\hline${ }^{74} \mathrm{Ge}(\mathrm{ppp}) \rightarrow{ }^{71} \mathrm{Cu}$ & N.A. & N.A. & 0.411 & 0.969 & 0.073 & 0.03 & 1.5 & 2 & 5.91 & 0.18 \\
\hline \multicolumn{11}{|c|}{ Decay-specific modes } \\
\hline${ }^{76} \mathrm{Ge}(\mathrm{ppp}) \rightarrow{ }^{73} \mathrm{Cu} e^{+} \pi^{+} \pi^{+}$ & 0.998 & 0.969 & 0.996 & 0.969 & 0.990 & 0.923 & 165. & 0 & 2.44 & 47.0 \\
\hline${ }^{76} \mathrm{Ge}(\mathrm{ppn}) \rightarrow{ }^{73} \mathrm{Zn} e^{+} \pi^{+}$ & 0.999 & 0.969 & 0.990 & N.A. & N.A. & 0.958 & 172. & 0 & 2.44 & 48.7 \\
\hline${ }^{76} \mathrm{Ge}(\mathrm{pp}) \rightarrow{ }^{74} \mathrm{Zn} \pi^{+} \pi^{+}$ & 0.994 & 0.968 & 0.972 & 0.964 & 0.991 & 0.893 & 160. & 0 & 2.44 & 45.5 \\
\hline${ }^{76} \mathrm{Ge}(\mathrm{pn}) \rightarrow{ }^{74} \mathrm{Ga} \pi^{0} \pi^{+}$ & 0.979 & 0.964 & 0.991 & N.A. & N.A. & 0.935 & 168. & 0 & 2.44 & 47.6 \\
\hline${ }^{74} \mathrm{Ge}(\mathrm{ppp}) \rightarrow{ }^{71} \mathrm{Cu} e^{+} \pi^{+} \pi^{+}$ & 0.998 & 0.969 & 0.993 & 0.969 & 0.982 & 0.912 & 46.6 & 0 & 2.44 & 13.2 \\
\hline${ }^{74} \mathrm{Ge}(\mathrm{ppn}) \rightarrow{ }^{71} \mathrm{Zn} e^{+} \pi^{+}$ & 0.999 & 0.967 & 0.982 & N.A. & N.A. & 0.949 & 48.5 & 0 & 2.44 & 13.8 \\
\hline${ }^{73} \mathrm{Ge}(\mathrm{ppp}) \rightarrow{ }^{70} \mathrm{Cu} e^{+} \pi^{+} \pi^{+}$ & 0.998 & 0.968 & 0.996 & N.A. & N.A. & 0.963 & 5.3 & 0 & 2.44 & 1.5 \\
\hline${ }^{73} \mathrm{Ge}(\mathrm{pnn}) \rightarrow{ }^{70} \mathrm{Ga} e^{+} \pi^{0}$ & 0.999 & 0.958 & 0.867 & N.A. & N.A. & 0.830 & 4.6 & 1 & 4.36 & 0.7 \\
\hline${ }^{73} \mathrm{Ge}(\mathrm{pp}) \rightarrow{ }^{71} \mathrm{Zn} \pi^{+} \pi^{+}$ & 0.994 & 0.967 & 0.982 & N.A. & N.A. & 0.944 & 5.2 & 0 & 2.44 & 1.5 \\
\hline${ }^{72} \mathrm{Ge}(\mathrm{ppp}) \rightarrow{ }^{69} \mathrm{Cu} e^{+} \pi^{+} \pi^{+}$ & 0.998 & 0.967 & 0.973 & N.A. & N.A. & 0.940 & 18.4 & 0 & 2.44 & 5.2 \\
\hline${ }^{72} \mathrm{Ge}(\mathrm{pn}) \rightarrow{ }^{70} \mathrm{Ga} \pi^{0} \pi^{+}$ & 0.979 & 0.958 & 0.867 & N.A. & N.A. & 0.813 & 16.0 & 1 & 4.36 & 2.5 \\
\hline${ }^{70} \mathrm{Ge}(\mathrm{nnn}) \rightarrow{ }^{67} \mathrm{Ge} \bar{\nu} \pi^{0}$ & 0.952 & 0.959 & 0.972 & N.A. & N.A. & 0.887 & 11.9 & 1 & 4.36 & 1.9 \\
\hline
\end{tabular}

\section{DECAY MODE SPECIFIC PROCESSES}

For decay modes specific to one of the processes in Eqs. (1) and (2), the signature benefits from the energy deposit of the initial decay process $\left(\epsilon_{0}\right)$ and the time correlation with the following decay of the unstable nucleus $\left(\epsilon_{\tau 1}\right)$. The decays in Eqs. (1) and (2) also have significant nuclear recoil kinetic energy, up to many 10 's of $\mathrm{MeV}$. A threshold of $11 \mathrm{MeV}$, chosen to lie above most of our events and near or at the digitizer saturation level, was applied to select these events. Even though edge effects can sometimes result in a modest lepton or pion energy deposit, the probability that the initial decay deposits more than $11 \mathrm{MeV}$ is over $95 \%$ for all decay channels.

We used MAGE to simulate these decay-mode-specific efficiencies also including all participating particles in Table III. The emitted particles deposit a great deal of energy for the considered decays. The phase space distribution of the n-body decay was calculated using the GENBOD function [32] in the TGenPhaseSpace class of ROOT [33]. As an example, Fig. 4 shows the phase space distribution for ${ }^{76} \mathrm{Ge} \rightarrow{ }^{73} \mathrm{Cu} e^{+} \pi^{+} \pi^{+}$. The efficiency was estimated as the fraction of the 10000 events with an energy larger than $11 \mathrm{MeV}$ deposited within the detector. The nuclear recoil energy included a correction for quenching using the Lindhard equation [34], but at these high energies, the shift in efficiency was less than the statistical uncertainty of the simulation, implying that quenching is not an important effect. Almost all events will have a large probability of saturating the detectors as shown in Table III. Due to this additional saturated event tag, the 2-MeV threshold constraint used for the invisible decay search can be relaxed. The energy threshold for the decayspecific modes is $100 \mathrm{keV}$, resulting in a significantly higher efficiency.

Therefore, there is a high probability that the event will be very distinctive. Although some saturated events arise from electrical breakdown and not physical processes, the associated waveforms are distinct from a saturated physics events and the two populations can be easily discerned by pulse shape analysis. In particular the onset of the waveform of a physics event is gradual, whereas for a breakdown it is a sharp upturn. Cosmic rays are also a source of saturated waveforms, but the veto system tags them efficiently.

For the decay-specific modes, we remove nonphysical waveforms but do not apply the DCR cut. The DCR cut is unnecessary because the saturated event trigger rate is very low, significantly reducing the background. For the decay-specific modes analyses, we also require full operation of the cosmic ray veto system as candidates will have a large energy deposit that is not muon induced. In DS0, the veto system was not fully implemented and we exclude that data from this analysis. This loss of exposure is accounted for in Table III. We then require energy and timing correlations between successive events, which differ from similar requirements for the invisible modes. 

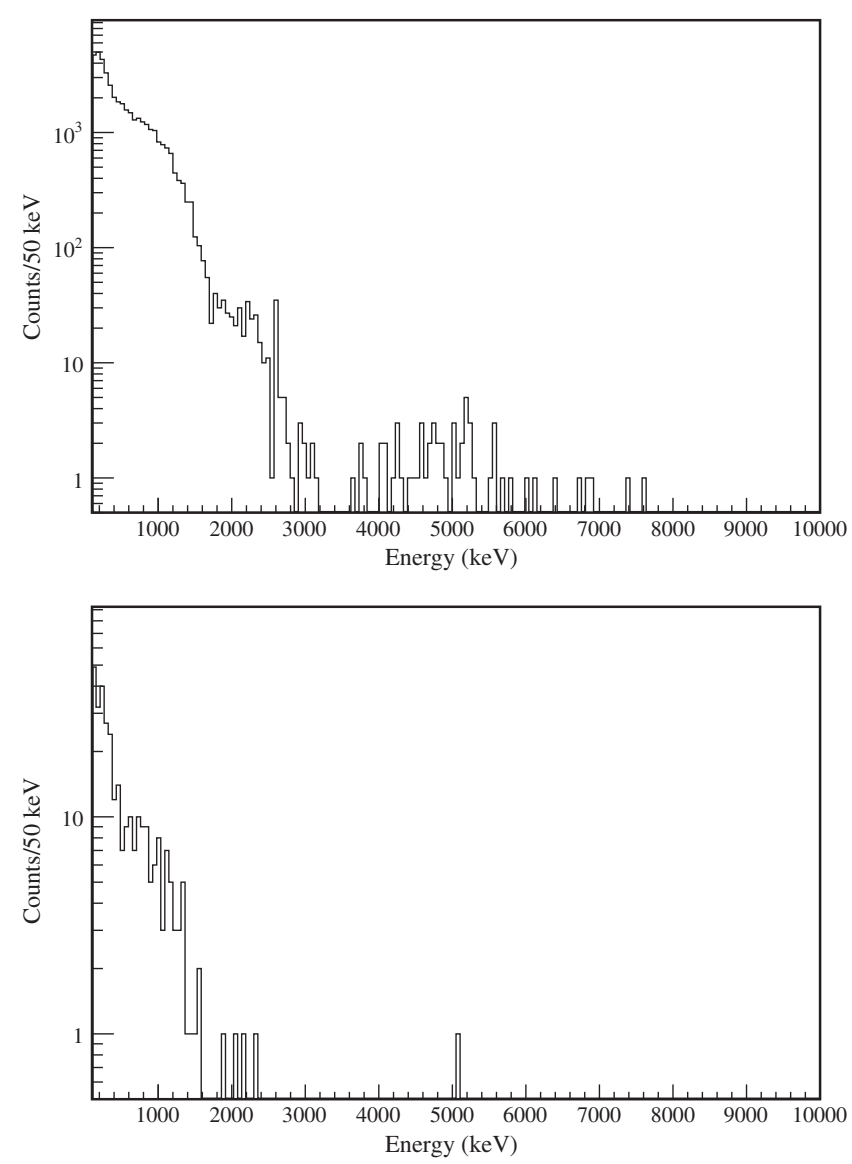

FIG. 3. Top: The spectrum of all events surviving after a muon veto cut and a DCR cut with energy greater than $100 \mathrm{keV}$ that follow a previous event with energy greater than $100 \mathrm{keV}$ in a given detector within a 40-minute delayed coincidence window. Bottom: The same as the top spectrum, except that the initial event is required to have at least $2 \mathrm{MeV}$, corresponding to one of the energy restrictions for candidates for invisible decay modes. Of the four events above $2 \mathrm{MeV}$, only two (described in Table II) meet the combined requirements of energy and time to be candidates.

The total efficiency $\left(\epsilon_{\text {tot }}\right)$ is equal to the product of all the efficiencies due to the time correlation cuts, the energy cuts, and the efficiency for the detection of the initial decay $\left(\epsilon_{0}\right)$. For the decay-specific modes, $\epsilon_{\mathrm{tot}}=$ $\epsilon_{0} \epsilon_{\tau 1} \epsilon_{\tau 2} \epsilon_{E 1} \epsilon_{E 2}$. Some processes we considered here only have one $\beta$ decay; in these cases, $\epsilon_{\tau 2}$ and $\epsilon_{E 2}$ are not applicable.

There is only one event with energy $>11 \mathrm{MeV}$ that meets the criteria to be a candidate. This event has a secondary energy deposition of $152 \mathrm{keV}$ that follows the saturated event by $75.7 \mathrm{~m}$. That event candidate matches the signature for three processes, ${ }^{73} \mathrm{Ge}(\mathrm{pnn}),{ }^{72} \mathrm{Ge}(\mathrm{pn})$, and ${ }^{70} \mathrm{Ge}(\mathrm{nnn})$, providing background for each. The other searched-for channels have zero candidates. The $T_{1 / 2}$ limits for 12 different decay-specific modes are listed in Table III.
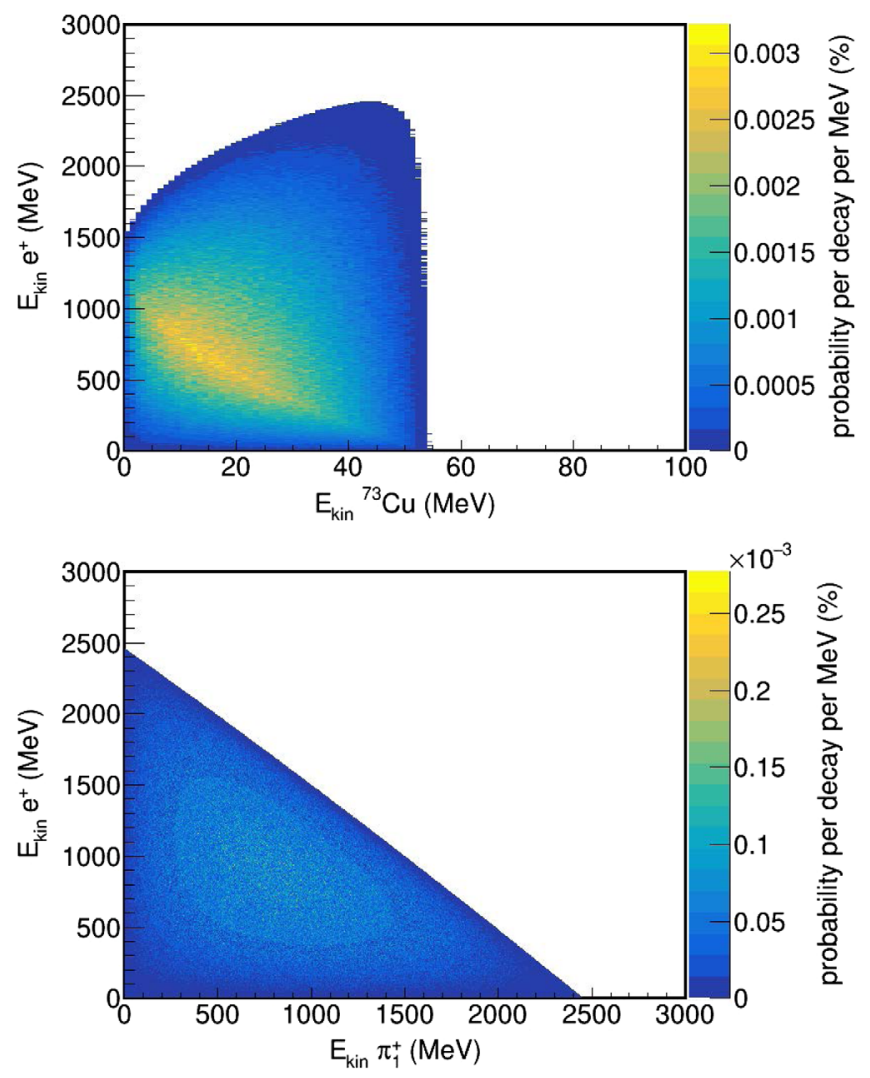

FIG. 4. The phase space distribution between particles in ${ }^{76} \mathrm{Ge} \rightarrow{ }^{73} \mathrm{Cu} e^{+} \pi^{+} \pi^{+}$.

\section{DISCUSSION}

The systematic uncertainties include the exposure uncertainty (2\%), uncertainty in the nonphysical event removal $(0.1 \%)$, uncertainty in the delayed charge recovery cut energy dependence (1\%), uncertainty due to how well the simulations model the detector $(2 \%)$, and the statistical uncertainty of the simulated efficiencies $(<1 \%)$. All of these are very small compared to the statistical uncertainty of $S$, and we ignore their contribution to the half-life limits. The simulations were for specific modes of decay and, hence, have that model dependency as an uncertainty; however, we quote limits for the specific modes simulated. We find no evidence for $\not \beta$ and the best limits for the various decay-specific modes are mid $10^{25} \mathrm{yr}$ range. The best limit for an invisible decay is for ${ }^{76} \mathrm{Ge}(\mathrm{ppp}) \rightarrow{ }^{73} \mathrm{Cu}$ with a half-life $>7.5 \times 10^{24} \mathrm{yr}$.

For the dinucleon modes, the Fréjus [6], KamLAND [10] and Super-Kamiokande [11-13] experiments have limits exceeding $10^{30} \mathrm{yr}$, reaching out to $4 \times 10^{32} \mathrm{yr}$. Neutronantineutron oscillations are also a $\Delta B=2$ test of $\not B$. SNO [35] reported a half-life limit for ${ }^{2} \mathrm{H}$ of $1.48 \times 10^{31} \mathrm{yr}$, and Super-Kamiokande [36] reported a half-life limit of $1.9 \times$ $10^{31} \mathrm{yr}$ for ${ }^{16} \mathrm{O}$. The DemOnstrator limits for dinulceon modes are much less restrictive than these previous efforts because of the lower exposure. We list the results, however, in case the nuclear dependence is of interest. 
It should be noted that some previous results are quoted in terms of a baryon half-life by attempting to account for the number of baryon combinations within a nucleus. Others quote a nuclear half-life. We chose the latter approach as the experimental result has less dependence on the model and interpretation. Furthermore, our quoted limits for each decay channel assume it is the dominant decay branch. This results in a conservative upper limit on the half-life for the considered channel. For example, ${ }^{73} \mathrm{Cu}$ could be populated by two-proton decay of ${ }^{76} \mathrm{Ge}$ to unbound states in ${ }^{74} \mathrm{Zn}$, which in turn emits a proton. This process would compete with the triproton decay of ${ }^{76} \mathrm{Ge}$. We neglect such side channels and quote the conservative lower value for the limit. It is also possible that the decay would result in excited states in ${ }^{73} \mathrm{Cu}$. In this case, the relaxation of this state would either be in coincidence with the initial decay products or would simply be a precursor event to our search. In neither case would that alter our search algorithm or efficiencies.

The best previous limits on $3 \mathrm{n}$ decays $\left(1.8 \times 10^{23} \mathrm{yr}\right)$ [5] come from a study in iodine, which also reported results for $4 \mathrm{n}$ decay $\left(1.4 \times 10^{23} \mathrm{yr}\right)$. This paper took account of the number of baryon combinations within the same shell orbit.

The Majorana Demonstrator provides an improved limit for $3 p$ invisible decay. The previous best limits on trinucleon decay come from EXO-200 [4] based on $223 \mathrm{~kg}$ yr of exposure. For the decay of ${ }^{136} \mathrm{Xe}(\mathrm{ppp}) \rightarrow{ }^{133} \mathrm{Sb}$, the limit is $3.3 \times 10^{23}$ yr. For ${ }^{136} \mathrm{Xe}(\mathrm{ppn}) \rightarrow{ }^{133} \mathrm{Te}$, the limit is $1.9 \times 10^{23} \mathrm{yr}$. The energy and time-coincidence cuts permit an event-by-event analysis in the DEMONSTRATOR, greatly reducing the background while maintaining a substantial efficiency. This results in an improved sensitivity over a spectral component fit approach.

\section{ACKNOWLEDGMENTS}

We thank Michael Graesser for discussions on baryon decay. This material is based upon work supported by the U.S. Department of Energy, Office of Science, Office of Nuclear Physics under Awards No. DE-AC02-05CH11231, No. DE-AC05-00OR22725, No. DE-AC05-76RL0130, No. DE-AC52-06NA25396, No. DE-FG02-97ER41020, No. DE-FG02-97ER41033, No. DE-FG02-97ER41041, No. DE-SC0010254, No. DE-SC0012612, No. DESC0014445, and No. DE-SC0018060. We acknowledge support from the Particle Astrophysics Program and Nuclear Physics Program of the National Science Foundation through Grants No. MRI-0923142, No. PHY-1003399, No. PHY-1102292, No. PHY1206314, No. PHY-1614611, No. PHY-1812409, and No. PHY-1812356. We gratefully acknowledge the support of the U.S. Department of Energy through the LANL/ LDRD Program and through the PNNL/LDRD Program for this work. We acknowledge support from the Russian Foundation for Basic Research, Grant No. 15-02-02919. We acknowledge the support of the Natural Sciences and Engineering Research Council of Canada, funding Reference No. SAPIN-2017-00023, and from the Canada Foundation for Innovation John R. Evans Leaders Fund. This research used resources provided by the Oak Ridge Leadership Computing Facility at Oak Ridge National Laboratory and by the National Energy Research Scientific Computing Center, a U.S. Department of Energy Office of Science User Facility. We thank our hosts and colleagues at the Sanford Underground Research Facility for their support.
[1] K. Babu et al., arXiv:1311.5285.

[2] K. Babu, I. Gogoladze, and K. Wang, Phys. Lett. B 570, 32 (2003).

[3] R. Bernabei et al., Eur. Phys. J. 27, 35 (2006).

[4] J. B. Albert et al., Phys. Rev. D 97, 072007 (2018).

[5] R. Hazama, H. Ejiri, K. Fushimi, and H. Ohsumi, Phys. Rev. C 49, 2407 (1994).

[6] C. Berger et al., Phys. Lett. B 269, 227 (1991).

[7] R. Bernabei et al., Phys. Lett. B 493, 12 (2000).

[8] H. Back et al., Phys. Lett. B 563, 23 (2003).

[9] V. Tretyak, V. Vu. Denisov, and Yu. G. Zdesenko, JETP Lett. 79, 106 (2004).

[10] T. Araki et al., Phys. Rev. Lett. 96, 101802 (2006).

[11] M. Litos et al., Phys. Rev. Lett. 112, 131803 (2014).

[12] V. Takhistov et al., Phys. Rev. Lett. 115, 121803 (2015).

[13] J. Gustafson et al., Phys. Rev. D 91, 072009 (2015).

[14] R. B. Firestone, V. S. Shirley, C. M. Baglin, S. Y. F. Chu, and J. Zipkin, Table of Isotopes (Springer, Hungary, 1997).
[15] N. Abgrall et al., Adv. High Energy Phys. 2014, 1 (2014).

[16] C. Aalseth et al., Phys. Rev. Lett. 120, 132502 (2018).

[17] J. Heise, J. Phys. Conf. Ser. 606, 012015 (2015).

[18] N. Abgrall et al., Phys. Rev. Lett. 118, 161801 (2017).

[19] S. I. Alvis et al., Phys. Rev. Lett. 120, 211804 (2018).

[20] N. Abgrall et al., Astropart. Phys. 93, 70 (2017).

[21] W. Bugg, Yu. Efremenko, and S. Vasilyev, Nucl. Instrum. Methods Phys. Res., Sect. A 758, 91 (2014).

[22] P. N. Luke, F. S. Goulding, N. W. Madden, and R. H. Pehl, IEEE Trans. Nucl. Sci. 36, 926 (1989).

[23] P. S. Barbeau, J. I. Collar, and O. Tench, J. Cosmol. Astropart. Phys. 09 (2007) 009.

[24] E. Aguayo et al., arXiv:1109.6913.

[25] R. J. Cooper, D. C. Radford, K. Lagergren, J. F. Colaresi, L. Darken, R. Henning, M. G. Marino, and K. M. Yocum, Nucl. Instrum. Methods Phys. Res., Sect. A 629, 303 (2011).

[26] S. Alvis et al., arXiv:1902.02299. 
[27] S. Agostinelli et al., Nucl. Instrum. Methods Phys. Res., Sect. A 506, 250 (2003).

[28] Y.-D. Chan et al., arXiv:0802.0860.

[29] M. Boswell et al., IEEE Trans. Nucl. Sci. 58, 1212 (2011).

[30] J. Gruszko et al., J. Phys. Conf. Ser. 888, 012079 (2017).

[31] G. J. Feldman and R. D. Cousins, Phys. Rev. D 57, 3873 (1998).
[32] F. E. James, Report No. CERN-68-15, CERN, Geneva, Switzerland 1968, https://cds.cern.ch/record/275743.

[33] R. Brun and F. Rademakers, Nucl. Instrum. Methods Phys. Res., Sect. A 389, 81 (1997).

[34] J. Lindhard, M. Scharff, and H. E. Schiott, Mat. Fys. Medd. K. Dan. Vidensk. Selsk 33, 14 (1963).

[35] B. Aharmim et al., Phys. Rev. D 96, 092005 (2017).

[36] K. Abe et al., Phys. Rev. D 91, 072006 (2015). 